\title{
A COMPACTNESS LEMMA AND ITS APPLICATION TO THE EXISTENCE OF MINIMIZERS FOR THE LIQUID DROP MODEL*
}

\author{
RUPERT L. FRANK ${ }^{\dagger}$ AND ELLIOTT H. LIEB ${ }^{\ddagger}$
}

\begin{abstract}
The ancient Gamow liquid drop model of nuclear energies has had a renewed life as an interesting problem in the calculus of variations: Find a set $\Omega \subset \mathbb{R}^{3}$ with given volume $A$ that minimizes the sum of its surface area and its Coulomb self energy. A ball minimizes the former and maximizes the latter, but the conjecture is that a ball is always a minimizer - when there is a minimizer. Even the existence of minimizers for this interesting geometric problem has not been shown in general. We prove the existence of the absolute minimizer (over all $A$ ) of the energy divided by $A$ (the binding energy per particle). A second result of our work is a general method for showing the existence of optimal sets in geometric minimization problems, which we call the "method of the missing mass." A third point is the extension of the pulling back compactness lemma [E. H. Lieb, Invent. Math., 74 (1983), pp. 441-448] from $W^{1, p}$ to $B V$.
\end{abstract}

Key words. liquid drop model, existence of minimizer, compactness

AMS subject classifications. 49Q10, 49Q20, 46T99, 49J40, 81V35

DOI. $10.1137 / 15 \mathrm{M} 1010658$

1. Introduction and main results. In this paper we are interested in some aspects of Gamow's famous 1930 "liquid drop model" [G]. This simple but successful model of atomic nuclei predicts, among other things,

(a) the spherical shape of nuclei,

(b) the nonexistence of nuclei with atomic number larger than some critical value, and

(c) the existence of a nucleus with minimal binding energy per particle.

Rather surprisingly, this model has received little attention in mathematics, and only recently have proofs appeared which rigorously derive properties (a) (partially) and (b). One of our goals in this paper will be to prove property (c). Before we describe these results in detail, we recall the model.

In the liquid drop model a nucleus $\Omega \subset \mathbb{R}^{3}$ is assumed to have constant density, which we may assume to be equal to one. Thus, $|\Omega|=A$ is the number of nucleons (protons and neutrons) in the nucleus. Mathematically, it is not necessary to assume that this number is an integer. The binding energy of a nucleus is given by

$$
\mathcal{E}(\Omega):=\operatorname{Per} \Omega+D(\Omega),
$$

where Per $\Omega$ denotes the surface area of $\Omega$, provided its boundary is smooth (see (2.1) for a definition of Per $\Omega$ for an arbitrary measurable set), and

$$
D(\Omega):=\frac{1}{2} \iint_{\Omega \times \Omega} \frac{d x d y}{|x-y|}
$$

\footnotetext{
* Received by the editors March 2, 2015; accepted for publication (in revised form) September 17, 2015; published electronically November 19, 2015.

http://www.siam.org/journals/sima/47-6/M101065.html

${ }^{\dagger}$ Mathematics 253-37, Caltech, Pasadena, CA 91125 (rlfrank@caltech.edu). The work of this author was partially supported by U.S. National Science Foundation PHY-1347399 and DMS-1363432.

$¥$ Departments of Mathematics and Physics, Princeton University, Princeton, NJ 08544 (lieb@ princeton.edu). The work of this author was partially supported by U.S. National Science Foundation PHY-1265118.
} 
denotes the Coulomb repulsion energy. We dropped a volume term $-e A$, where $e$ is the energy of "nuclear matter" per unit volume in the "thermodynamic limit." (This energy is computed using only the short-range strong forces, without the Coulomb repulsion.) This term only contributes a constant in our case and need not be exhibited explicitly for our considerations, but stability of a real nucleus requires its inclusion; the total energy must be negative. The surface term Per $\Omega$ accounts for corrections to the volume term coming from the surface of the nucleus and acts as a surface tension. The Coulomb term $D(\Omega)$ describes the repulsion between the protons in the nucleus. The charge (which we set equal to one in the definition of $D(\Omega)$ ) is an effective charge proportional to the ratio of the proton number to $A$. We also neglected asymmetry and pairing terms.

The minimal binding energy of a nucleus with mass number $A>0$ is given by

$$
E(A):=\inf \{\mathcal{E}(\Omega):|\Omega|=A\} .
$$

The following is rigorously known about the properties (a) and (b) mentioned before.

(a) There is an $A_{c_{1}}>0$ such that $E(A)$ is minimized by a ball for all $0<A \leq A_{c_{1}}$ and such that a ball does not minimize $E(A)$ for $A>A_{c_{1}}$. It is even known that balls are the unique minimizers for $A<A_{c_{1}}$.

(b) There is an $A_{c_{2}}>0$ such that $E(A)$ has no minimizer for $A>A_{c_{2}}$.

Fact (a) was proved by Knüpfer and Muratov [KM2, Thm. 3.2] (balls are minimizers for $A \leq A_{c_{1}}$; see also $[\mathrm{J}]$ ) and by Bonacini and Cristoferi [BC, Thm. 2.10] (balls are unique minimizers for $A<A_{c_{1}}$ and are not minimizers for $A>A_{c_{1}}$ ). Fact (b) is proved by Knüpfer and Muratov [KM2, Thm. 3.3] and by Lu and Otto [LO, Thm. 2] and their 2014 presentation of this work at the Fields Institute was partial motivation for our interest in the problem.

Fact (b) has the physical interpretation that nuclei "fission" spontaneously when they are too large. When the mass is slightly below the critical value $A_{c_{2}}$ they are still unstable against being impacted by a neutron - with the release of some notable amount of energy. In this model the reduction in energy is electrostatic (Coulomb), not nuclear, so one might properly refer to a "Coulomb bomb" rather than to a "nuclear bomb."

It is conjectured $[\mathrm{CP}]$, but not known, that $A_{c_{1}}=A_{c_{2}}$. (For some analogous results in related models we refer the reader to [KM1, MZ, BC].)

In this paper we show the following (see Theorem 3.2):

(c) There is a set $\Omega_{*} \subset \mathbb{R}^{3}$ such that

$$
\frac{E(A)}{A} \geq \frac{\mathcal{E}\left(\Omega_{*}\right)}{\left|\Omega_{*}\right|}
$$

for any $A>0$.

Physically, the set $\Omega_{*}$ corresponds to a nucleus with the greatest binding energy per particle. This is ${ }^{62} \mathrm{Ni}$, but some theoretical calculations indicate that the highest value should occur for $A=58.3, Z=26.6[\mathrm{~F}]$. Our proof of (c) makes use of the Knüpfer-Muratov and Lu-Otto analyses in [KM2] and [LO]. From (c) one can easily deduce that (see Corollary 3.3)

$$
\lim _{A \rightarrow \infty} \frac{E(A)}{A}=\frac{\mathcal{E}\left(\Omega_{*}\right)}{\left|\Omega_{*}\right|} .
$$

Recall from (b) that

$$
A_{c_{2}}:=\sup \{A>0: E(A) \text { has a minimizer }\}<\infty .
$$


We consider the question whether there is a minimizer for $E\left(A_{c_{2}}\right)$. By definition there is a sequence of sets $\Omega_{n}$ with $A_{n}:=\left|\Omega_{n}\right| \rightarrow A_{c_{2}}$ which minimize $E\left(A_{n}\right)$. The question is how these sets behave as $n \rightarrow \infty$. If they have a limit, this limit is a good candidate for a minimizer for $E\left(A_{c_{2}}\right)$. On the other hand, the fact that there is no minimizer beyond $A_{c_{2}}$ means, in some sense, that the Coulomb energy becomes the dominant term at $A_{c_{2}}$. In order to minimize the Coulomb energy, one might think that the sets $\Omega_{n}$ become more and more elongated, which could cause the failure of a nontrivial limit. That this possible scenario does not occur is our next result (see Theorem 3.4).

(d) $E\left(A_{c_{2}}\right)$ has a minimizer.

Physically, a corresponding minimizing set $\Omega_{c_{2}}$ corresponds to lead $(\mathrm{Pb})$, a stable nucleus with the largest possible nucleon number. Our proof of (d) uses, crucially, bounds from [KM2, LO] for the proof of (b).

We also prove the following (physically obvious) fact (see Theorem 3.1).

(e) If for a given $A>0$ one has the strict binding inequality $E(A)<E\left(A^{\prime}\right)+$ $E\left(A-A^{\prime}\right)$ for all $0<A^{\prime}<A$, then there is a minimizer for $E(A)$.

We emphasize that the strict binding inequality is only a sufficient condition for the existence of a minimizer. For instance, in the situation of (d) it is probably violated.

A second goal in this paper, in addition to the study of the liquid drop model, is to present a method to prove the existence of optimal sets in geometric minimization problems that involve the perimeter functional. We believe that our method is simpler and more direct than methods used, for instance, in [dPV] and has applications in related problems.

The method that we are using may be called the method of the missing mass and originates in the solution of the Hardy-Littlewood-Sobolev (HLS) problem [L2] and is also used in the paper [BN, Lemma 1.2 and Appendix]. In this method each element of a minimizing sequence is decomposed into two terms, namely, a main piece with good convergence properties and a missing piece which vanishes in a weak sense. The key point is then to use this missing piece as a potential minimizer. Typically, this allows one to conclude that the limit of the main piece is a minimizer. This method is often, but not in this paper, combined with a strengthening of Fatou's lemma which includes the missing piece; see [L2] and, for an extension to a wide class of convex functions, see [BL1]. (The latter paper also contains a review of how this method is used in [L2] and $[\mathrm{BN}]$.)

The method of the missing mass works both in problems with and without translation invariance. The minimization problem in $[\mathrm{BN}]$ is not translation invariant, and the translation invariance in [L2] is broken by symmetric decreasing rearrangement. In situations with translation invariance, however, as in the present paper, there is the additional difficulty of detecting a main piece which has a nonzero limit. This problem was overcome in [L3]; see [BL2, FLL] for early applications of this pulling back compactness lemma to concrete minimization problems. More recent applications include $[\mathrm{FL}, \mathrm{BFV}]$.

As far as we know, the method of the missing mass has not been applied to geometric minimization problems in which the minimization is over sets. In this paper we identify a possible nonzero limit after translations in much the same way as in [L3], where the case of the Sobolev space $W^{1, p}\left(\mathbb{R}^{d}\right)$ with $p>1$ was treated. Our case here corresponds to $p=1$, where the space $B V\left(\mathbb{R}^{d}\right)$ of functions of bounded variation has to be used to get better compactness properties; see Proposition 2.1. What is considerably more involved than in the $p=2$ case is to split the elements of the minimizing sequence into a main piece and a missing piece. This is accomplished 
in Lemma 2.2, and the method used has some similarities with the one used in [FLSS] in a different physical context.

2. Compactness up to translations. In this section we describe sequences of subsets of $\mathbb{R}^{d}$ with a uniform perimeter bound, and we prove a pulling back compactness theorem, which extends that of [L3, sect. 3] to functions of bounded variation.

If $E_{n}$ and $E$ are measurable sets in $\mathbb{R}^{d}$, we say that $E_{n} \rightarrow E$ (globally) if $\left|E_{n} \Delta E\right| \rightarrow 0$, and we say that $E_{n} \rightarrow E$ locally if for every compact set $K \subset \mathbb{R}^{d}$,

$$
\left|\left(E_{n} \Delta E\right) \cap K\right| \rightarrow 0 .
$$

If $E \subset \mathbb{R}^{d}$ is a measurable set, we denote by

$$
\text { Per } E=\sup \left\{\int_{E} \operatorname{div} F d x: F \in C_{0}^{1}\left(\mathbb{R}^{d}, \mathbb{R}^{d}\right),|F| \leq 1\right\}
$$

its perimeter in the sense of De Giorgi. Moreover, $B_{r}(a)$ denotes the open ball of radius $r$ centered at $a \in \mathbb{R}^{d}$, and we abbreviate $B_{r}=B_{r}(0)$.

The following proposition is our technical main result. As explained in the introduction, its proof relies on a technique from [L3].

Proposition 2.1. Let $\left(E_{n}\right)$ be a sequence of measurable sets in $\mathbb{R}^{d}$ with uniformly bounded perimeter. Then one of the following two alternatives occurs:

1. $\lim _{n \rightarrow \infty}\left|E_{n}\right|=0$.

2. There is a set $E$ with positive measure and a sequence $\left(a_{k}\right)$ in $\mathbb{R}^{d}$ such that for a subsequence $\left(n_{k}\right)$ one has

$$
E_{n_{k}}-a_{k} \rightarrow E \quad \text { locally. }
$$

Moreover, $|E| \leq \liminf _{k \rightarrow \infty}\left|E_{n_{k}}\right|$ and $\operatorname{Per} E \leq \liminf _{k \rightarrow \infty} \operatorname{Per} E_{n_{k}}$.

Proof. We begin by proving that for every $u \in W^{1,1}\left(\mathbb{R}^{d}\right)$ and every $r>0$,

$$
\int_{\mathbb{R}^{d}}\left(|\nabla u|+C r^{-1}|u|\right) d x \geq c\left(\sup _{a \in \mathbb{R}^{d}}\left|B_{r}(a) \cap\{u \neq 0\}\right|\right)^{-1 / d} \int_{\mathbb{R}^{d}}|u| d x
$$

for some constants $c$ and $C$ depending only on $d$. In fact, let $\chi$ be a smooth function with support in $B_{1}$ and $\|\chi\|_{1}=1$. We put $\chi_{a, r}(x)=r^{-d} \chi((x-a) / r)$ and compute

$$
\int_{\mathbb{R}^{d}}\left|\nabla\left(\chi_{a, r} u\right)\right| d x \leq \int_{\mathbb{R}^{d}}\left(\left|\chi_{a, r}\right||\nabla u|+\left|\nabla \chi_{a, r}\right||u|\right) d x .
$$

On the other hand, by a Sobolev inequality and a Hölder inequality,

$$
\begin{aligned}
\int_{\mathbb{R}^{d}}\left|\nabla\left(\chi_{a, r} u\right)\right| d x & \geq c\left(\int_{\mathbb{R}^{d}}\left|\chi_{a, r} u\right|^{d /(d-1)} d x\right)^{(d-1) / d} \\
& \geq c\left(\sup _{a \in \mathbb{R}^{d}}\left|B_{r}(a) \cap\{u \neq 0\}\right|\right)^{-1 / d} \int_{\mathbb{R}^{d}}\left|\chi_{a, r} u\right| d x .
\end{aligned}
$$

When we integrate both the upper and the lower bounds on $\left\|\nabla\left(\chi_{a, r} u\right)\right\|_{1}$ with respect to $a$, we obtain $(2.2)$ with $C=\|\nabla \chi\|_{1}$.

By a standard argument [AFP, Thm. 3.9] (2.2) extends to $u \in B V\left(\mathbb{R}^{d}\right)$ and therefore, in particular, to $u=\chi_{E_{n}}$, where $\left(E_{n}\right)$ has uniformly bounded perimeter. For $r=1$, we find that

$$
\sup _{a \in \mathbb{R}^{d}}\left|B_{1}(a) \cap E_{n}\right| \geq\left(\frac{c\left|E_{n}\right|}{\operatorname{Per} E_{n}+C\left|E_{n}\right|}\right)^{d} .
$$


If we assume that alternative 1 does not occur, we may pass to a subsequence and assume that $\inf _{n}\left|E_{n}\right|>0$. Thus the right side of the previous inequality is bounded away from zero, and so there are $a_{n} \in \mathbb{R}^{d}$ such that

$$
\inf _{n}\left|B_{1} \cap\left(E_{n}-a_{n}\right)\right|=\inf _{n}\left|B_{1}\left(a_{n}\right) \cap E_{n}\right|>0 .
$$

We now apply the compactness result for sets of finite perimeter [AFP, Thm. 3.39] to $E_{n}-a_{n}$. We infer that there is a set $E$ of finite perimeter such that $E_{n}-a_{n} \rightarrow E$ locally. It is easy to see that local convergence implies that $\lim \inf \left|E_{n}\right|=\lim \inf \mid E_{n}-$ $a_{n}|\geq| E \mid$. Finally, lower semicontinuity of the perimeter, that is, $\lim \inf \operatorname{Per} E_{n}=$ $\operatorname{Per}\left(E_{n}-a_{n}\right) \geq \operatorname{Per} E$, follows easily from the variational definition of the perimeter.

Next, we describe sequences of sets which converge locally but not globally, because they lose measure in the limit. We show that such sets can be decomposed into a main piece, which does converge globally, and a remainder piece, which disappears from any compact set.

LEMMA 2.2. Let $\left(E_{n}\right)$ be a sequence of measurable sets in $\mathbb{R}^{d}$ with finite perimeter such that $E_{n} \rightarrow E$ locally for some set $E \subset \mathbb{R}^{d}$. Assume that $0<|E|<$ $\liminf \operatorname{in}_{n \rightarrow \infty}\left|E_{n}\right|$. Then there is a sequence $\left(r_{n}\right)$ in $(0, \infty)$ such that the sets

$$
F_{n}:=E_{n} \cap B_{r_{n}}, \quad G_{n}:=E_{n} \cap\left(\mathbb{R}^{d} \backslash \overline{B_{r_{n}}}\right)
$$

satisfy

$$
\lim _{n \rightarrow \infty}\left(\operatorname{Per} E_{n}-\operatorname{Per} F_{n}-\operatorname{Per} G_{n}\right)=0
$$

and

$$
F_{n} \rightarrow E \text { (globally) and } \quad G_{n} \rightarrow \emptyset \text { locally }
$$

In particular,

$$
\lim _{n \rightarrow \infty}\left|F_{n}\right|=|E| \quad \text { and } \quad \liminf _{n \rightarrow \infty} \operatorname{Per} F_{n} \geq \operatorname{Per} E .
$$

Proof. Clearly, we have

$$
\text { Per } E_{n} \leq \operatorname{Per} F_{n}+\operatorname{Per} G_{n} \leq \operatorname{Per} E_{n}+2 \sigma_{r_{n}}\left(\partial B_{r_{n}} \cap E_{n}\right),
$$

where $\sigma_{r}$ denotes surface measure on the sphere $\partial B_{r}$. Thus, for the bound (2.3) on the perimeter, we have to prove that

$$
\sigma_{r_{n}}\left(\partial B_{r_{n}} \cap E_{n}\right) \rightarrow 0
$$

for a suitable choice of $r_{n}$.

In order to construct the $r_{n}$, we distinguish two cases. We first assume that $E$ is (essentially) bounded, say, $E \subset B_{R}$ for some $R>0$. Then $E_{n} \rightarrow E$ locally implies that $\varepsilon_{n}:=\left|E_{n} \cap\left(B_{2 R} \backslash B_{R}\right)\right| \rightarrow 0$. We claim that for every $n$ there is an $r_{n} \in[R, 2 R]$ with $\sigma_{r_{n}}\left(\partial B_{r_{n}} \cap E_{n}\right) \leq \varepsilon_{n} / R$, proving (2.6). In fact, if this were not the case, we would have, by integration in spherical coordinates,

$$
\varepsilon_{n}<\int_{R}^{2 R} \sigma_{r}\left(\partial B_{r} \cap E_{n}\right) d r=\left|E_{n} \cap\left(B_{2 R} \backslash B_{R}\right)\right|=\varepsilon_{n},
$$


which is a contradiction.

Now assume that $E$ is (essentially) unbounded, and define $R_{n}$ by $\left|E_{n} \cap B_{R_{n}}\right|=|E|$. (This is well defined if $\left|E_{n}\right|>|E|$, which we may assume after discarding finitely many $E_{n}$ 's.) We claim that $R_{n} \rightarrow \infty$. In fact, if we had $R_{n_{k}} \rightarrow R_{*}$ for some $R_{*}<\infty$, then, using $E_{n} \rightarrow E$ locally,

$$
|E|=\left|E_{n_{k}} \cap B_{R_{n_{k}}}\right|=\left|E_{n_{k}} \cap B_{R_{*}}\right|+o(1)=\left|E \cap B_{R_{*}}\right|+o(1)<|E|+o(1),
$$

which is a contradiction. Now choose $R$ such that $\left|E \cap B_{R}\right|=|E| / 2$. Then, since $E_{n} \rightarrow E$ locally, for all sufficiently large $n,\left|E_{n} \cap B_{R}\right| \geq|E| / 4$. After discarding finitely many $n$ we may assume that $R_{n}>R$. We claim that for every $n$ there is an $r_{n} \in\left[\left(R+R_{n}\right) / 2, R_{n}\right]$ with $\sigma_{r_{n}}\left(\partial B_{r_{n}} \cap E_{n}\right) \leq 3|E| /\left(2\left(R_{n}-R\right)\right)$, proving (2.6) once more. In fact, if this were not the case, we would have, like before,

$\frac{3}{4}|E|<\int_{\frac{R+R_{n}}{2}}^{R_{n}} \sigma_{r}\left(\partial B_{r} \cap E_{n}\right) d r=\left|E_{n} \cap\left(B_{R_{n}} \backslash B_{\frac{R+R_{n}}{2}}\right)\right| \leq\left|E_{n} \cap B_{R_{n}}\right|-\left|E_{n} \cap B_{R}\right| \leq \frac{3}{4}|E|$,

which is a contradiction. This completes the proof of (2.3).

It remains to prove (2.4) (which implies (2.5)), and, to do so, we again distinguish two cases. Assume first that $E$ is bounded, and note that

$$
\left|F_{n} \Delta E\right|=2\left|F_{n} \backslash E\right|+|E|-\left|F_{n}\right| .
$$

Since $F_{n} \supset E_{n} \cap B_{R}$, we have $\left|F_{n}\right| \geq\left|E_{n} \cap B_{R}\right|$ and, by local convergence, $\left|E_{n} \cap B_{R}\right| \rightarrow$ $\left|E \cap B_{R}\right|=|E|$. Thus, liminf $\left|F_{n}\right| \geq|E|$. On the other hand, we have $F_{n} \subset E_{n} \cap B_{2 R}$, and therefore $\left|F_{n} \backslash E\right| \leq\left|\left(E_{n} \backslash E\right) \cap B_{2 R}\right|$. By local convergence, $\left|\left(E_{n} \backslash E\right) \cap B_{2 R}\right| \rightarrow 0$. Because of (2.7) we conclude that $\left|F_{n} \Delta E\right| \rightarrow 0$. Finally, if $K$ is compact, then $\left|G_{n} \cap K\right| \leq\left|E_{n} \cap\left(\mathbb{R}^{d} \backslash \overline{B_{R}}\right) \cap K\right|$. By local convergence, the latter converges to $\left|E \cap\left(\mathbb{R}^{d} \backslash \overline{B_{R}}\right) \cap K\right|=0$, so we conclude that $G_{n} \rightarrow \emptyset$ locally.

Now assume that $E$ is unbounded, and note that

$$
\left|F_{n} \Delta E\right|=2\left|E \backslash F_{n}\right|+\left|F_{n}\right|-|E| .
$$

Since $F_{n} \subset E_{n} \cap B_{R_{n}}$, we have $\left|F_{n}\right| \leq\left|E_{n} \cap B_{R_{n}}\right|=|E|$. On the other hand, we have $\left|E \backslash F_{n}\right|=\left|\left(E \backslash E_{n}\right) \cap B_{r_{n}}\right|+\left|E \backslash B_{r_{n}}\right|$. By dominated convergence, since $r_{n} \rightarrow \infty$, we have $\left|E \backslash B_{r_{n}}\right| \rightarrow 0$. Now, given $\varepsilon>0$, let $\rho>0$ such that $\left|E \cap\left(\mathbb{R}^{d} \backslash \overline{B_{\rho}}\right)\right| \leq \varepsilon$. Then, if $r_{n} \geq \rho,\left|\left(E \backslash E_{n}\right) \cap B_{r_{n}}\right|=\left|\left(E \backslash E_{n}\right) \cap B_{\rho}\right|+\left|\left(E \backslash E_{n}\right) \cap B_{r_{n}} \cap\left(\mathbb{R}^{d} \backslash \overline{B_{\rho}}\right)\right|$. We have $\left|\left(E \backslash E_{n}\right) \cap B_{\rho}\right| \rightarrow 0$ by local convergence and $\left|\left(E \backslash E_{n}\right) \cap B_{r_{n}} \cap\left(\mathbb{R}^{d} \backslash \overline{B_{\rho}}\right)\right| \leq \varepsilon$. All this proves that $\left|E \backslash F_{n}\right| \rightarrow 0$. Because of (2.8) we conclude that $\left|F_{n} \Delta E\right| \rightarrow 0$. Finally, if $K$ is compact, then $K \cap\left(\mathbb{R}^{d} \backslash B_{r_{n}}\right)=\emptyset$ for all sufficiently large $n$, so $\left|G_{n} \cap K\right|=0$, and we conclude that $G_{n} \rightarrow \emptyset$ locally. This concludes the proof of the lemma.

Clearly, the sets $F_{n}$ and $G_{n}$ from the previous proposition satisfy

$$
\left|F_{n}\right|+\left|G_{n}\right|=\left|E_{n}\right| \text {. }
$$

We next show that, at least asymptotically, some nonlocal functionals are also additive with respect to this decomposition. For $0<\lambda<d$ let

$$
I_{\lambda}(E)=\frac{1}{2} \iint_{E \times E} \frac{d x d y}{|x-y|^{\lambda}}
$$

Lemma 2.3. Let $F_{n}$ and $G_{n}$ be sequences of measurable sets in $\mathbb{R}^{d}$ with uniformly bounded measure, and $\left|F_{n} \cap G_{n}\right|=0$ for all $n$. Assume that

$$
F_{n} \rightarrow E \text { (globally) and } \quad G_{n} \rightarrow \emptyset \text { locally }
$$


for some set $E$. Then for all $0<\lambda<d$,

$$
I_{\lambda}\left(F_{n} \cup G_{n}\right)=I_{\lambda}\left(F_{n}\right)+I_{\lambda}\left(G_{n}\right)+o(1)
$$

and

$$
I_{\lambda}\left(F_{n}\right)=I_{\lambda}(E)+o(1) .
$$

Proof. As a preliminary to the proof we note that for an arbitrary set $F \subset \mathbb{R}^{d}$ of finite measure, by a simple rearrangement inequality [LL, Thm. 3.4],

$$
\int_{F} \frac{d y}{|x-y|^{\lambda}} \leq \int_{F^{*}} \frac{d y}{|y|^{\lambda}}=C|F|^{(d-\lambda) / d}
$$

with some explicit $C$ depending only on $d$ and $\lambda$. Here $F^{*}$ denotes the ball centered at the origin with the same measure as $F$. Moreover,

$$
\int_{F} \frac{d y}{|x-y|^{\lambda}} \rightarrow 0 \quad \text { as }|x| \rightarrow \infty .
$$

(This follows from [LL, Thm. 2.2] by decomposing $|x|^{-\lambda}$ in a short-range part and a long-range part.)

We now turn to the proof of (2.9). We need to show that

$$
2 I_{\lambda}\left(F_{n}, G_{n}\right):=\iint_{F_{n} \times G_{n}} \frac{d x d y}{|x-y|^{\lambda}} \rightarrow 0 .
$$

Using (2.11) and $F_{n} \rightarrow E$, we find

$$
\left|I_{\lambda}\left(F_{n}, G_{n}\right)-I_{\lambda}\left(E, G_{n}\right)\right| \leq I_{\lambda}\left(F_{n} \Delta E, G_{n}\right) \leq C\left|F_{n} \Delta E\right|^{(d-\lambda) / d}\left|G_{n}\right| \rightarrow 0,
$$

so it is enough to prove $I_{\lambda}\left(E, G_{n}\right) \rightarrow 0$. Let $\varepsilon>0$ be given, and use (2.12) to find $R>0$ such that $\int_{E}|x-y|^{-\lambda} d y \leq \varepsilon$ for $|x| \geq R$. We decompose and bound

$I_{\lambda}\left(E, G_{n}\right)=I_{\lambda}\left(E, G_{n} \cap B_{R}\right)+I_{\lambda}\left(E, G_{n} \backslash B_{R}\right) \leq C|E|^{(d-\lambda) / d}\left|G_{n} \cap B_{R}\right|+\varepsilon\left|G_{n} \backslash B_{R}\right|$.

Since $G_{n} \rightarrow \emptyset$ locally, we have $\left|G_{n} \cap B_{R}\right| \rightarrow 0$, and therefore $I_{\lambda}\left(E, G_{n}\right) \rightarrow 0$, as claimed. The proof of (2.10) is similar.

\section{Application to the liquid drop model.}

3.1. Results. In this section we return to the liquid drop model discussed in the introduction. Thus, we assume $d=3$ and $\lambda=1$ and abbreviate $I_{1}(\Omega)=D(\Omega)$. We recall that the binding energy of a set $\Omega$ and the minimal binding energy with nucleon number $A$ were defined as

$$
\mathcal{E}(\Omega)=\operatorname{Per} \Omega+D(\Omega) \quad \text { and } \quad E(A)=\inf \{\mathcal{E}(\Omega):|\Omega|=A\} .
$$

It is easy to see (and is proved, for instance, in [LO]) that

$$
E(A) \leq E\left(A^{\prime}\right)+E\left(A-A^{\prime}\right) \quad \text { for all } 0<A^{\prime}<A .
$$

Our first theorem states that if this inequality is strict, then there is a minimizer for $E(A)$.

Theorem 3.1. Let $A>0$ such that

$$
E(A)<E\left(A^{\prime}\right)+E\left(A-A^{\prime}\right) \quad \text { for all } 0<A^{\prime}<A \text {. }
$$


Then the infimum defining $E(A)$ is attained. Moreover, any minimizing sequence has a subsequence which, after a translation, converges (globally) to a minimizer.

We recall that (global) convergence of sets was defined at the beginning of section 2. It means that the measure of the symmetric difference between the sets and their limit tends to zero.

Of physical interest is the quantity

$$
e(A):=\frac{E(A)}{A}
$$

the binding energy per particle.

Theorem 3.2. There is an $A_{*}>0$ such that $e\left(A_{*}\right)=\inf \{e(A): A>0\}$. Moreover, the infimum defining $E\left(A_{*}\right)$ is attained.

In other words, there is a set $\Omega_{*} \subset \mathbb{R}^{3}$ with finite perimeter such that

$$
\frac{\operatorname{Per} \Omega+D(\Omega)}{|\Omega|} \geq \frac{\operatorname{Per} \Omega_{*}+D\left(\Omega_{*}\right)}{\left|\Omega_{*}\right|} \quad \text { for all } \Omega \subset \mathbb{R}^{3} \text { with finite perimeter. }
$$

Theorem 3.2 has the following simple corollary.

Corollary 3.3. As $A \rightarrow \infty, e(A) \rightarrow e\left(A_{*}\right)$. Moreover, $e\left(k A_{*}\right)=e\left(A_{*}\right)$ for all $k \in \mathbb{N}$.

Proof of Corollary 3.3. Subadditivity (3.1) and nonnegativity of $E(A)$ imply by abstract principles that $\lim _{A \rightarrow \infty} e(A)$ exists. (See, e.g., [HP, Thm. 7.6.1]; this is sometimes called "Fekete's lemma.") Iterating (3.1), we infer that $e\left(k A_{*}\right) \leq e\left(A_{*}\right)$ for all $k \in \mathbb{N}$. On the other hand, since $A_{*}$ is the global minimum of $e(A)$, we have $e\left(k A_{*}\right) \geq e\left(A_{*}\right)$. So $e\left(k A_{*}\right)=e\left(A_{*}\right)$ for all $k \in \mathbb{N}$, and, since we know that $e(A)$ has a limit, this limit must be equal to $e\left(A_{*}\right)$, as claimed.

Our final theorem is as follows.

Theorem 3.4. The set $\{A>0: E(A)$ has a minimizer $\}$ is closed.

This proves, in particular, that for $A_{c_{2}}=\sup \{A>0: E(A)$ has a minimizer $\}$ there is a minimizer.

3.2. Proofs of Theorems 3.1, 3.2, and 3.4. We now prove our three main theorems about the liquid drop model.

Proof of Theorem 3.1. Let $\left(\Omega_{n}\right)$ with $\left|\Omega_{n}\right|=A$ be a minimizing sequence for $E(A)$. Since Per $\Omega_{n} \leq \mathcal{E}\left(\Omega_{n}\right)=E(A)+o(1)$ is uniformly bounded, Proposition 2.1 yields a set $\Omega \subset \mathbb{R}^{3}$ with $0<|\Omega| \leq A$ and $\operatorname{Per} \Omega \leq \lim$ inf Per $\Omega_{n}$ such that, after passing to a subsequence and a translation, we have $\Omega_{n} \rightarrow \Omega$ locally. Moreover, by Fatou's lemma, we have $D(\Omega) \leq \liminf D\left(\Omega_{n}\right)$, so $\mathcal{E}(\Omega) \leq \liminf \mathcal{E}\left(\Omega_{n}\right)$. Thus, $\Omega$ will be a minimizer provided we can show that $|\Omega|=A$. Moreover, it is easy to see that $\Omega_{n} \rightarrow \Omega$ locally and $\left|\Omega_{n}\right| \rightarrow|\Omega|$ implies that $\Omega_{n} \rightarrow \Omega$ globally. Therefore, both statements of the theorem follow if we can prove that $|\Omega|=A$.

We argue by contradiction and assume that $|\Omega|<A$. Then, by Lemma 2.2, we can write $\Omega_{n}=F_{n} \cup G_{n}, F_{n} \cap G_{n}=\emptyset$, such that

$$
\operatorname{Per} \Omega_{n} \geq \operatorname{Per} \Omega+\operatorname{Per} G_{n}+o(1) \text {. }
$$

Moreover, $F_{n} \rightarrow \Omega$ globally and $G_{n} \rightarrow \emptyset$ locally, which, by Lemma 2.3 , implies that

$$
D\left(\Omega_{n}\right)=D(\Omega)+D\left(G_{n}\right)+o(1) .
$$


Thus,

$$
\begin{aligned}
E(A) & =\mathcal{E}\left(\Omega_{n}\right)+o(1) \\
& \geq \mathcal{E}(\Omega)+\mathcal{E}\left(G_{n}\right)+o(1) \\
& \geq E(|\Omega|)+E\left(\left|G_{n}\right|\right)+o(1)
\end{aligned}
$$

Since $\left|G_{n}\right|=\left|\Omega_{n}\right|-\left|F_{n}\right| \rightarrow A-|\Omega|$ and since $A \mapsto E(A)$ is continuous on $(0, \infty)$ (in fact, $A^{-2 / 3} E(A)=\inf \{\operatorname{Per} \omega+A D(\omega):|\omega|=1\}$ is concave as an infimum over affine linear functions), we obtain in the limit $n \rightarrow \infty$

$$
E(A) \geq E(|\Omega|)+E(A-|\Omega|)
$$

which contradicts assumption (3.2). Thus, $|\Omega|=A$.

We prove Theorem 3.2 via the auxiliary minimization problem

$$
e_{\leq}(A):=\inf \left\{\frac{\mathcal{E}(\Omega)}{|\Omega|}: 0<|\Omega| \leq A\right\} .
$$

The idea of relaxing the equality constraint $|\Omega|=A$ to $|\Omega| \leq A$ is reminiscent of [LS].

Lemma 3.5. For any $A>0$, the infimum defining $e_{\leq}(A)$ is attained.

Proof. Let $\left(\Omega_{n}\right)$ with $\left|\Omega_{n}\right| \leq A$ be a minimizing sequence for $e_{\leq}(A)$. By the isoperimetric inequality, we have $e_{\leq}(A)+o(1)=\mathcal{E}\left(\Omega_{n}\right) /\left|\Omega_{n}\right| \geq C\left|\Omega_{n}\right|^{-1 / 3}$, which implies that $\lim \inf \left|\Omega_{n}\right|>0$. Moreover, $\operatorname{Per} \Omega_{n} \leq \mathcal{E}\left(\Omega_{n}\right) \leq A\left(e_{\leq}(A)+o(1)\right)$ is uniformly bounded, so, as in the proof of Theorem 3.1, we obtain, after passing to subsequence and after a translation, a set $\Omega \subset \mathbb{R}^{3}$ with $0<|\Omega| \leq A$ and with $\Omega_{n} \rightarrow \Omega$ locally and $\mathcal{E}(\Omega) \leq \liminf \mathcal{E}\left(\Omega_{n}\right)$. We now distinguish two cases according to how $|\Omega|$ compares to $\liminf \left|\Omega_{n}\right|$.

If $|\Omega| \geq \liminf \left|\Omega_{n}\right|$, then $\mathcal{E}(\Omega) /|\Omega| \leq \lim \sup \left(\mathcal{E}\left(\Omega_{n}\right) /\left|\Omega_{n}\right|\right)=e_{\leq}(A)$, so $\Omega$ is a minimizer for $e_{\leq}(A)$.

If $|\Omega|<\lim$ inf $\left|\Omega_{n}\right|$, then, as in the proof of Theorem 3.1,

$$
e_{\leq}(A)=\frac{\mathcal{E}(\Omega)+\mathcal{E}\left(G_{n}\right)}{|\Omega|+\left|G_{n}\right|}+o(1) \geq \frac{\mathcal{E}(\Omega)}{|\Omega|+\left|G_{n}\right|}+\frac{\left|G_{n}\right|}{|\Omega|+\left|G_{n}\right|} e_{\leq}(A)+o(1) .
$$

Rearranging the terms, we obtain $|\Omega| e_{\leq}(A) \geq \mathcal{E}(\Omega)+o(1)$, which again means that $\Omega$ is a minimizer for $e_{\leq}(A)$.

Proof of Theorem 3.2. Clearly, $A \mapsto e_{\leq}(A)$ is nonincreasing and nonnegative, and it is continuous because of the continuity of $E \mapsto E(A)$ (see the proof of Theorem 3.1). Let

$$
A_{*}:=\sup \left\{A>0: \text { there is an } A^{\prime}>A \text { with } e_{\leq}\left(A^{\prime}\right)<e_{\leq}(A)\right\} .
$$

We claim that $A_{*}<\infty$. Clearly, this implies that $e_{\leq}(A)=e_{\leq}\left(A_{*}\right)$ for all $A \geq A_{*}$ and therefore that $e(A) \geq e\left(A_{*}\right)$ for all $A>0$. Moreover, the minimizer $\Omega_{*}$ for $e_{\leq}\left(A_{*}\right)$, which exists by Lemma 3.5, has $\left|\Omega_{*}\right|=A_{*}$ and therefore is also a minimizer for $\bar{E}\left(A_{*}\right)$.

We argue by contradiction and assume that $A_{*}=\infty$; that is, there is an increasing sequence $\left(A_{n}\right)$ with $A_{n} \rightarrow \infty$ such that $e_{\leq}\left(A_{n+1}\right)<e_{\leq}\left(A_{n}\right)$ for all $n$. According to Lemma 3.5, there are sets $\Omega_{n}$ with $\mathcal{E}\left(\Omega_{n}\right)=e_{\leq}\left(A_{n}\right)\left|\Omega_{n}\right|$ and $\left|\Omega_{n}\right| \leq A_{n}$. The strict inequality $e_{\leq}\left(A_{n+1}\right)<e_{\leq}\left(A_{n}\right)$ implies that $\left|\Omega_{n+1}\right|>A_{n}$, and so, in particular, $A_{n}^{\prime}:=\left|\Omega_{n}\right| \rightarrow \infty$. Since the sets $\Omega_{n}$ minimize $e_{\leq}\left(A_{n}^{\prime}\right)$, they also minimize $E\left(A_{n}^{\prime}\right)$, but the existence of minimizers for this problem with arbitrarily large $A_{n}^{\prime}$ contradicts 
the result in [KM2, Thm. 3.3] and [LO, Thm. 2]. This proves that $A_{*}<\infty$, as claimed.

Remark 3.6. The minimization problem of Theorem 3.2 is equivalent to the following scale-invariant minimization problem:

$$
I=\inf \left\{\frac{(\operatorname{Per} \Omega)^{2 / 3} D(\Omega)^{1 / 3}}{|\Omega|}: \Omega \subset \mathbb{R}^{3} \text { of finite perimeter }\right\} .
$$

(We know from [KM2, Lem. 7.1] that $I>0$.) In fact, in order to minimize $\mathcal{E}(\Omega) /|\Omega|$, we can minimize separately over shape and size of $\Omega=\ell \omega$; that is,

$$
\inf _{\Omega} \frac{\mathcal{E}(\Omega)}{|\Omega|}=\inf _{|\omega|=1} \inf _{\ell>0}\left(\ell^{-1} \operatorname{Per} \omega+\ell^{2} D(\omega)\right) .
$$

For fixed $\omega$, the infimum is attained at $\ell_{\omega}=\left(\operatorname{Per} \omega /(2 D(\omega))^{1 / 3}\right.$, and we have

$$
\inf _{\Omega} \frac{\mathcal{E}(\Omega)}{|\Omega|}=2^{-2 / 3} \cdot 3 \cdot \inf _{|\omega|=1}(\operatorname{Per} \omega)^{2 / 3} D(\omega)^{1 / 3}=2^{-2 / 3} \cdot 3 I .
$$

In particular, if $\omega$ has $|\omega|=1$ and minimizes $(\operatorname{Per} \Omega)^{2 / 3} D(\omega)^{1 / 3}$, then $\Omega:=\ell_{\omega} \omega$ minimizes $\mathcal{E}(\Omega) /|\Omega|$.

Proof of Theorem 3.4. Let $A_{n}$ be a sequence with $A_{n} \rightarrow A \in(0, \infty)$ such that $E\left(A_{n}\right)$ has a minimizer $\Omega_{n}$ with $\left|\Omega_{n}\right|=A_{n}$. As observed in the proof of Theorem 3.1, $A \mapsto E(A)$ is continuous, so $E\left(A_{n}\right) \rightarrow E(A)$ and Per $\Omega_{n}$ is uniformly bounded. As in the proof of Theorem 3.1, we may assume that $\Omega_{n} \rightarrow \Omega$ locally for a set $\Omega \subset \mathbb{R}^{3}$ with $0<|\Omega| \leq A$ and $\mathcal{E}(\Omega) \leq \liminf \mathcal{E}\left(\Omega_{n}\right)$, and it remains to prove that $|\Omega|=A$.

To prove this, we use a bound from [KM2] and [LO]. Namely, for every $\varepsilon>0$ there is a constant $C_{\varepsilon}>0$ such that if $A^{\prime} \geq \varepsilon$ and $\Omega^{\prime} \subset \mathbb{R}^{3}$ is a minimizer for $E\left(A^{\prime}\right)$, then

$$
\operatorname{diam} \Omega^{\prime} \leq C_{\varepsilon} A
$$

This is stated in [KM2, Lemma 7.2] with $\varepsilon=1$, but the same proof works for $\varepsilon<1$. (Technically speaking, in order to avoid ambiguities with sets of measure zero, $\Omega^{\prime}$ is replaced by the set $\left\{x \in \mathbb{R}^{3}: \limsup _{r \rightarrow 0}\left|\Omega^{\prime} \cap B_{r}(x)\right| /\left|B_{r}(x)\right|>0\right\}$. By Lebesgue's differentiation theorem, this set coincides with $\Omega^{\prime}$ up to sets of measure zero, so none of the terms in the minimization problem change under this replacement.)

Applying (3.4) to $\Omega^{\prime}=\Omega_{n}$, we infer that $\lim \sup _{n \rightarrow \infty} \operatorname{diam} \Omega_{n}<\infty$. Thus, the sets $\Omega_{n}$ are contained in a fixed ball, and then local convergence $\Omega_{n} \rightarrow \Omega$ implies global convergence, so $|\Omega|=\lim \left|\Omega_{n}\right|=\lim A_{n}=A$, as claimed.

Remark 3.7. Theorems 3.1 and 3.4 remain true, mutatis mutandis, for the functional $\mathcal{E}(\Omega)=\operatorname{Per} \Omega+I_{\lambda}(\Omega), \Omega \subset \mathbb{R}^{d}$, with $d \geq 2$ and $0<\lambda<d$. (For the analogue of the diameter bound (3.4), see [KM2, Lemma 7.2].) Theorem 3.2 and Corollary 3.3 remain true under the additional assumption $0<\lambda<2$. (Under this assumption the analogue of the nonexistence result remains valid; see [KM2, Thm. 3.3].)

\section{Further remarks about the liquid drop model.}

4.1. A lower bound on $\boldsymbol{A}_{*}$. Let us consider the binding energy per particle for balls,

$$
e^{(b a l l)}(A):=A^{-1 / 3} \frac{\operatorname{Per} B}{|B|^{2 / 3}}+A^{2 / 3} \frac{D(B)}{|B|^{5 / 3}},
$$


where $B$ is the unit ball in $\mathbb{R}^{3}$. (So $|B|=4 \pi / 3$, Per $B=4 \pi$, and $D(B)$ can be computed, but we do not need this.) Clearly, $e^{(\text {ball })}(A)$ decreases up to some $A_{*}^{(\text {ball })}>$ 0 and then increases. Setting the $A$-derivative of $e^{(\text {ball })}(A)$ equal to zero, we find

$$
A_{*}^{(\text {ball })}=\frac{|B| \operatorname{Per} B}{2 D(B)} .
$$

The main result of this section is the following quantitative bound on the curve $e(A)$.

Proposition 4.1. Let

$$
A_{0}:=\sup \left\{A_{0}>0: A \mapsto e(A) \text { is strictly decreasing on }\left(0, A_{0}\right)\right\} \text {. }
$$

Then $A_{0} \geq A_{*}^{(\text {ball })}$ with equality iff $E\left(A_{0}\right)$ is minimized by a ball.

Note that this gives, in particular, the lower bound $A_{*} \geq A_{*}^{(b a l l)}$ on the number $A_{*}$ from Theorem 3.2.

Proof. We know from [KM2] that $E(A)$ is minimized by balls for small $A$, and therefore $e(A)$ is decreasing for small $A$, so $A_{0}>0$. From Theorem 3.2 we know that $e(A)$ has a global minimum, so $A_{0}<\infty$. By assumption we have $e(A)>e\left(A_{0}\right)$ for all $0<A<A_{0}$, and, therefore,

$$
E\left(A^{\prime}\right)+E\left(A_{0}-A^{\prime}\right)>A^{\prime} e\left(A_{0}\right)+\left(A_{0}-A^{\prime}\right) e\left(A_{0}\right)=E\left(A_{0}\right) \quad \text { for all } 0<A^{\prime}<A_{0} .
$$

According to Theorem 3.1, this implies that there is a minimizer $\Omega_{0}$ for $E\left(A_{0}\right)$.

The next step is to derive a virial relation for $\Omega_{0}$. Since $e(A)$ has a local minimum at $A_{0}$, the function

$$
\ell \mapsto \frac{\mathcal{E}\left(\ell \Omega_{0}\right)}{\left|\ell \Omega_{0}\right|}=\ell^{-1} \frac{\operatorname{Per} \Omega_{0}}{\left|\Omega_{0}\right|}+\ell^{2} \frac{D\left(\Omega_{0}\right)}{\left|\Omega_{0}\right|}
$$

has a local minimum at $\ell=1$. Setting the derivative at $\ell=1$ equal to zero, we conclude that

$$
\operatorname{Per} \Omega_{0}=2 D\left(\Omega_{0}\right)
$$

We now use (4.3) to prove a lower bound on $A_{0}$. For the proof we will use the inequalities

$$
\text { Per } E \geq \operatorname{Per} E^{*} \quad \text { and } \quad D\left(E^{*}\right) \geq D(E)
$$

where $E^{*}$ denotes a ball of the same measure as $E$. The first inequality is just a rewriting of the isoperimetric inequality, and the second one follows, for instance, from the Riesz rearrangement inequality [LL, Thm. 3.7]. From these inequalities and (4.3) we deduce that

$$
D\left(\Omega_{0}\right)=\frac{1}{2} \operatorname{Per} \Omega_{0} \geq \frac{1}{2} \operatorname{Per} \Omega_{0}^{*}=\frac{1}{2} \frac{\operatorname{Per} B}{D(B)^{2 / 5}} D\left(\Omega_{0}^{*}\right)^{2 / 5} \geq \frac{1}{2} \frac{\operatorname{Per} B}{D(B)^{2 / 5}} D\left(\Omega_{0}\right)^{2 / 5} .
$$

(The middle equality here just uses scaling.) Thus,

$$
D\left(\Omega_{0}\right)^{3 / 5} \geq \frac{1}{2} \frac{\operatorname{Per} B}{D(B)^{2 / 5}} .
$$


On the other hand, again by (4.4),

$$
D\left(\Omega_{0}\right)^{3 / 5} \leq D\left(\Omega_{0}^{*}\right)^{3 / 5}=\frac{D(B)^{3 / 5}}{|B|}\left|\Omega_{0}^{*}\right|=\frac{D(B)^{3 / 5}}{|B|}\left|\Omega_{0}\right| .
$$

(The middle equality again just uses scaling.) Combining the last two inequalities and recalling (4.2), we obtain

$$
\left|\Omega_{0}\right| \geq \frac{|B| \operatorname{Per} B}{2 D(B)}=A_{*}^{(\text {ball })}
$$

as claimed.

Finally, assume that $\Omega_{0}$ is not a ball. Then the strict rearrangement inequality $D\left(\Omega_{0}^{*}\right)>D\left(\Omega_{0}\right)[\mathrm{L} 1]$ implies, by the same argument as before, the strict inequality $\left|\Omega_{0}\right|>A_{*}^{(b a l l)}$. This concludes the proof.

Remark 4.2. The same proof shows that the virial relation (4.3) holds whenever $A_{0}$ is a local minimum of $e(A)$ and $E\left(A_{0}\right)$ is attained by some $\Omega_{0}$.

4.2. Dissociation into balls. Let us consider

$$
\tilde{e}(A):=A^{-1} \inf \{\mathcal{E}(\Omega):|\Omega|=A, \Omega \text { is a countable union of disjoint balls }\} .
$$

This is the same as the energy $e(A)$ per particle except that we restrict the allowed sets to be countable disjoint unions of balls. Clearly, it suffices to consider the case when the individual balls are infinitely far apart, and therefore

$$
\tilde{e}(A)=\inf \left\{\sum_{k=1}^{\infty} \frac{A_{k}}{A} e^{(\text {ball })}\left(A_{k}\right): \sum_{k=1}^{\infty} A_{k}=A\right\}
$$

with the energy $e^{(\text {ball })}\left(A_{k}\right)$ from (4.1). It turns out that this minimization problem can be almost explicitly solved.

Proposition 4.3. For any $A>0$,

$$
\tilde{e}(A)=\min _{k \in \mathbb{N}} e^{(\text {ball })}(A / k)
$$

That is, $\tilde{e}(A)$ coincides with the energy per particle of $K$ infinitely separated balls of volume $A / K$, where $K$ is to be optimized over. The energy per particle of such a ball satisfies $e^{(\text {ball })}(A / K) \sim\left(|\partial B| /\left(A^{1 / 3}|B|^{2 / 3}\right)\right) K^{1 / 3}$ as $K \rightarrow \infty$, and therefore the infimum over $K$ is indeed attained at some finite $K$. With a little more work one can compute explicit numbers $0=a_{0}<a_{1}<a_{2}<a_{3}<\ldots$ with $a_{k} \rightarrow \infty$ such that $\tilde{e}(A)=e^{(b a l l)}(A / k)$ for $a_{k-1} \leq A \leq a_{k}$. For instance,

$$
a_{1}=\frac{2-2^{2 / 3}}{2^{2 / 3}-1} \frac{|B| \operatorname{Per} B}{D(B)} .
$$

The proof of Proposition 4.3 is based on the following technical lemma.

Lemma 4.4. Let $\lambda>0$ and $f(\theta)=\left(\theta^{2 / 3}+(1-\theta)^{2 / 3}\right)+\lambda\left(\theta^{5 / 3}+(1-\theta)^{5 / 3}\right)$. Then, for all $\theta \in[0,1]$,

$$
f(\theta) \geq \min \{f(0), f(1 / 2), f(1)\}
$$

with strict inequality unless $\theta \in\{0,1 / 2,1\}$. 
Proof of Lemma 4.4. Differentiating, we find

$$
f^{\prime}(\theta)=\frac{5 \lambda}{3}\left(\theta^{-1 / 3}-(1-\theta)^{-1 / 3}\right)\left(\frac{2}{5 \lambda}-g(\theta)\right),
$$

where

$$
g(\theta):=\frac{(1-\theta)^{2 / 3}-\theta^{2 / 3}}{\theta^{-1 / 3}-(1-\theta)^{-1 / 3}} .
$$

Clearly, $g$ is symmetric with respect to $\theta=1 / 2$ and satisfies $g(0)=g(1)=0$. By straightforward differentiation we find that $g$ is strictly concave, so it attains its maximum at $\theta=1 / 2$, and we compute $g(1 / 2)=1$. Thus for every $0 \leq \gamma<1$ there is a unique $\theta(\gamma) \in[0,1 / 2)$ such that $g(\theta)=\gamma$ iff $\theta= \pm \theta(\gamma)$.

Returning with this information to (4.5), we find that, if $2 /(5 \lambda) \geq 1$, then $f^{\prime}$ has a single zero at $\theta=1 / 2$, and, if $2 /(5 \gamma)<1$, then $f^{\prime}$ has three zeros $\theta=1 / 2, \pm \theta(2 /(5 \lambda))$. Since $f$ is clearly increasing near 0 and decreasing near 1 , the single zero of $f^{\prime}$ at $\theta=1 / 2$ for $2 /(5 \lambda) \geq 1$ and the two zeros at $\theta= \pm \theta(2 /(5 \lambda))$ for $2 /(5 \lambda)<1$ must correspond to local maxima of $f$. Thus, $f$ attains its minimum at $\theta \in\{0,1\}$ for $2 /(5 \lambda) \geq 1$. For $2 /(5 \lambda)<1, f$ has a local minimum at $\theta=1 / 2$, and it attains its minimum on a subset of $\{0,1 / 2,1\}$. This proves the lemma.

Proof of Proposition 4.3. We need only prove the inequality $\geq$. We first note that in the definition of $\tilde{e}(A)$ we can restrict ourselves to finite sums. Indeed, for any $K \in \mathbb{N}$, put $\tilde{A}_{K}=\sum_{k=K}^{\infty} A_{k}$, so that

$$
\sum_{k=1}^{\infty} \frac{A_{k}}{A} e^{(\text {ball })}\left(A_{k}\right) \geq\left(\sum_{k=1}^{K-1} \frac{A_{k}}{A} e^{(\text {ball })}\left(A_{k}\right)+\frac{\tilde{A}_{K}}{A} e^{(\text {ball })}\left(\tilde{A}_{K}\right)\right)-\frac{\tilde{A}_{K}}{A} e^{(\text {ball })}\left(\tilde{A}_{K}\right) .
$$

Since $a e^{(b a l l)}(a) \rightarrow 0$ as $a \rightarrow 0$, the last term goes to zero as $K \rightarrow \infty$. The first term can be bounded from below by the claimed expression if we prove the result for finite sums, as we will do now.

In fact, we will prove that

$$
\inf \left\{\sum_{k=1}^{K} \frac{A_{k}}{A} e^{(\text {ball })}\left(A_{k}\right): \sum_{k=1}^{K} A_{k}=A\right\}=\min _{K \geq k \in \mathbb{N}} e^{(\text {ball })}(A / k)
$$

by induction over $K \in \mathbb{N}$. For $K=1$ (4.6) is clearly true. Now assume that $K \geq 2$ and that the assertion is proved for $1, \ldots, K-1$. The set $\left\{\left(A_{1}, \ldots, A_{K}\right) \in[0, A]^{K}\right.$ : $\left.\sum A_{k}=A\right\}$ is compact, and the function to be minimized on the left side of (4.6) is continuous (note again that $a e^{(b a l l)}(a)$ is continuous at $a=0$ ), so there is a minimizer $A^{(0)}$. If $A_{k}^{(0)}=0$ for some $k$, then (4.6) follows by the induction assumption. Therefore, we may assume that $A_{k}^{(0)} \neq 0$ for all $k$. Let $1 \leq i<j \leq K$ be arbitrary. We shall show that $A_{i}^{(0)}=A_{j}^{(0)}$, which will prove (4.6). The contribution from these two numbers to the left side in (4.6) is

$$
\begin{aligned}
\frac{A_{i}^{(0)}}{A} e^{(\text {ball })}\left(A_{i}^{(0)}\right)+\frac{A_{j}^{(0)}}{A} e^{(\text {ball })}\left(A_{j}^{(0)}\right)= & \left(\theta^{2 / 3}+(1-\theta)^{2 / 3}\right)\left(A_{i}^{(0)}+A_{j}^{(0)}\right)^{2 / 3} \frac{P(B)}{A|B|^{2 / 3}} \\
& +\left(\theta^{5 / 3}+(1-\theta)^{5 / 3}\right)\left(A_{i}^{(0)}+A_{j}^{(0)}\right)^{5 / 3} \frac{D(B)}{A|B|^{5 / 3}}
\end{aligned}
$$


with $\theta=A_{i}^{(0)} /\left(A_{i}^{(0)}+A_{j}^{(0)}\right)$. By construction we have $\theta \neq 0,1$. If we had $\theta \neq 1 / 2$, then by Lemma 4.4 this contribution would be strictly less than for $\theta=1 / 2$. Thus, replacing both $A_{i}^{(0)}$ and $A_{j}^{(0)}$ by $\left(A_{i}^{(0)}+A_{j}^{(0)}\right) / 2$, we would get a strictly smaller energy while preserving the constraint, but this contradicts the minimality of $A^{(0)}$. Thus, we have $\theta=1 / 2$, that is, $A_{i}^{(0)}=A_{j}^{(0)}$, which is what we wanted to prove.

Note added in proof. After our paper was submitted, a preprint by Knüpfer, Muratov, and Novaga appeared $[\mathrm{KMN}]$ with similar results but with a different methodology. Some of those results had been mentioned by Muratov at the Fields Center meeting in 2014. We are grateful to C. Muratov for helpful remarks on a previous version of this paper.

\section{REFERENCES}

[AFP] L. Ambrosio, N. Fusco, and D. Pallara Functions of Bounded Variation and Free Discontinuity Problems, Oxford Mathematical Monographs, Oxford University Press, New York, 2000.

[BFV] J. Bellazzini, R. L. Frank, and N. Visciglia, Maximizers for Gagliardo-Nirenberg inequalities and related non-local problems, Math. Ann., 360 (2014), pp. 653-673.

[BC] M. BONACINI AND R. CRISTOFERI, Local and global minimality results for a nonlocal isoperimetric problem on $\mathbb{R}^{N}$, SIAM J. Math. Anal., 46 (2014), pp. 2310-2349.

[BL1] H. BRÉZIS AND E. H. LIEB, A relation between pointwise convergence of functions and convergence of functionals, Proc. Amer. Math. Soc., 88 (1983), pp. 486-490.

[BL2] H. BrÉzis AND E. H. Lieb, Minimum action solutions of some vector field equations, Comm. Math. Phys., 96 (1984), pp. 97-113.

[BN] H. Brézis And L. Nirenberg, Positive solutions of nonlinear elliptic equations involving critical Sobolev exponents, Comm. Pure Appl. Math., 36 (1983), pp. 437-477.

[CP] R. Choksi ANd M. A. Peletier, Small volume-fraction limit of the diblock copolymer problem: II. Diffuse-interface functional, SIAM J. Math. Anal., 43 (2011), pp. 739763.

[dPV] G. DE Philippis And B. Velichkov, Existence and regularity of minimizers for some spectral functionals with perimeter constraints, Appl. Math. Optim., 69 (2014), pp. 199231.

[F] M. P. Fewell, The atomic nuclide with the highest mean binding energy, Amer. J. Phys., 63 (1995), pp. 653-658.

[FL] R. L. Frank AND E. H. Lieb, Sharp constants in several inequalities on the Heisenberg group, Ann. of Math. (2), 176 (2012), pp. 349-381.

[FLSS] R. L. Frank, E. H. Lieb, R. Seiringer, and H. Siedentop, Müller's exchange-correlation energy in density-matrix-functional theory, Phys. Rev. A, 76 (2007), 052517.

[FLL] J. Fröhlich, E. H. Lieb, AND M. Loss, Stability of Coulomb systems with magnetic fields. I. The one-electron atom, Comm. Math. Phys., 104 (1986), pp. 251-270.

[G] G. Gamow, Mass defect curve and nuclear constitution, Proc. R. Soc. London Ser. A, 126 (1930), pp. 632-644.

[HP] E. Hille AND R. S. Phillips, Functional Analysis and Semi-Groups, Amer. Math. Soc. Colloq. Publ. 31, AMS, Providence, RI, 1957.

[J] V. Julin, Isoperimetric problem with a Coulomb repulsive term, Indiana Univ. Math. J., 63 (2014), pp. 77-89.

[KM1] H. KNÜPFer And C. B. Muratov, On an isoperimetric problem with a competing non-local term. I. The planar case, Comm. Pure Appl. Math., 66 (2013), pp. 1129-1162.

[KM2] H. KNÜPFER AND C. B. Muratov, On an isoperimetric problem with a competing nonlocal term II: The general case, Comm. Pure Appl. Math., 67 (2014), pp. 1974-1994.

[KMN] H. KNüPfer, C. B. Muratov, and M. Novaga, Low density phases in a uniformly charged liquid, preprint, arXiv:1504.05600, 2015.

[L1] E. H. LIEB, Existence and uniqueness of the minimizing solution of Choquard's nonlinear equation, Stud. Appl. Math., 57 (1976/1977), pp. 93-105.

[L2] E. H. LieB, Sharp constants in the Hardy-Littlewood-Sobolev and related inequalities, Ann. of Math. (2), 118 (1983), pp. 349-374.

[L3] E. H. LIEB, On the lowest eigenvalue of the Laplacian for the intersection of two domains, Invent. Math., 74 (1983), pp. 441-448. 
[LL] E. H. Lieb And M. Loss, Analysis, 2nd ed., Grad. Stud. Math. 14. AMS, Providence, RI, 2001.

[LS] E. H. Lieb And B. Simon, The Thomas-Fermi theory of atoms, molecules and solids, Adv. Math., 23 (1977), pp. 22-116.

[LO] J. Lu And F. OTto, Nonexistence of minimizers for Thomas-Fermi-Dirac-von Weizsäcker model, Comm. Pure Appl. Math., 67 (2014), pp. 1605-1617.

[MZ] C. B. Muratov And A. ZAleski, On an isoperimetric problem with a competing non-local term: Quantitative results, Ann. Global Anal. Geom., 47 (2014), pp. 63-80. 